\title{
Novel anti-infective potential of salvianolic acid $B$ against human serious pathogen Neisseria meningitidis
}

\author{
Sanna Huttunen ${ }^{1,2}$, Marko Toivanen ${ }^{1}$, Chenghai Liu ${ }^{3}$ and Carina Tikkanen-Kaukanen ${ }^{4^{*}}$
}

\begin{abstract}
Background: Epidemics of meningococcal meningitis cause significant health problems especially in Sub-Saharan Africa. Novel anti-infective candidates are needed. In modern anti-adhesion therapy initial attachment of bacteria to host cells is prevented. Our unique studies have revealed anti-adhesive candidates from natural products, namely milk and berries, against Neisseria meningitidis adhesion. In the present study against N. meningitidis adhesion, a novel binding inhibitor was found; salvianolic acid B (SA-B), a polyphenol from the radix Salviae miltiorrhizae, an important part of Chinese folk medicine.

Methods: In order to test inhibition of meningococcal pili binding and anti-adhesion activity of SA-B, bovine thyroglobulin, a reference glycoprotein for meningococcal receptor was used in a microtiter plate assay. Inhibitory activity was tested by using serial dilutions of SA-B extracts of 98 and $70 \%$ purity. Results were confirmed in a HEC-1B cell dot assay and antimicrobial activity was measured by using a microbroth dilution assay.

Results: Almost total (93 \%) inhibition of pili binding, anti-adhesion, was achieved with the $70 \%$ extract of SA-B at the concentration of $0.3 \mathrm{mg} / \mathrm{mL}$ in the bovine thyroglobulin reference model. $50 \%$ binding inhibition activity was achieved with $0.6 \mu \mathrm{g} / \mathrm{mL}$ of the SA-B extract. Total inhibition of the pili binding to HEC-1B cells was found at the tested concentration of $0.5 \mathrm{mg} / \mathrm{mL}$. The $98 \%$ pure SA-B resulted in weaker inhibition. At the concentration of $0.3 \mathrm{mg} /$ $\mathrm{mL} 78 \%$ inhibition was achieved in the thyroglobulin model. For $50 \%$ inhibition $2.4 \mu \mathrm{g} / \mathrm{mL}$ of pure SA-B was needed. The difference between the binding inhibition activities ( 70 and $98 \%$ pure $S A-B)$ was statistically significant $(P=0.03)$. Antimicrobial activity of $70 \%$ SA-B, when investigated against N. meningitidis, was detected only in relatively high concentrations.
\end{abstract}

Conclusions: Our results indicate that plant SA-B may prevent meningococcal infections by inhibiting meningococcal binding and may thus have an impact on the amount of nasopharyngeal carriers of $N$. meningitidis. This may prevent the spreading of meningococcal infections between humans. One could conclude that SA-B and its source dried radix S. miltiorrhizae, which is an important part of Chinese folk medicine, could be valuable candidates for further research in meningococcal disease prevention.

Keywords: Anti-adhesion therapy, Antimicrobial, Neisseria meningitidis, Salvianolic acid B, Polyphenol

\section{Background}

Due to increasing bacterial resistance it is important to search for new means against bacterial infections [1]. In anti-adhesion therapy the adhesion of bacteria to host

*Correspondence: carina.tikkanen-kaukanen@helsinki.fi

${ }^{4}$ Ruralia Institute, University of Helsinki, Lönnrotinkatu 7, 50170 Mikkeli,

Finland

Full list of author information is available at the end of the article tissues is inhibited by soluble carbohydrates or their analogs [1-3]. Based on animal experiments soluble carbohydrates can protect against experimental infections [4]. Studies with natural products: milk, berries, berry polyphenols and herbal medicines have indicated these products being possible sources for anti-adhesive agents [5-14]. 
Neisseria meningitidis, which is an important human pathogen, colonizing the nasopharynx of 10-35\% of young adults, can be transmitted from person to person by droplet infection [15]. For a small amount of the colonised people meningococci can cause life-threatening infections, such as meningococcal septicaemia or meningitis [16]. Epidemics of meningitis are a significant health problem especially in Sub-Saharan Africa [17] but also amongst risk groups such as in military forces. The attachment of $N$. meningitidis to human mucosal epithelial cells, the crucial step of the infection [18], is mediated by type IV pili [19]. HEC-1B epithelial cell line [9, 19] and bovine thyroglobulin [5] have been previously used for adhesion and binding, as well as functioned as binding inhibition models for meningococcal pili.

Our previous studies have shown that in the thyroglobulin model oligosaccharides isolated from human and bovine milk have anti-adhesion activity against $N$. meningitidis [5]. We have also shown that in microtiter well binding and cell culture inhibition assays polyphenolic fractions extracted from berries possess anti-adhesive activity against $N$. meningitidis $[6,9]$.

In the present study we tested anti-adhesion and antimicrobial activities of salvianolic acid B (SA-B) (Fig. 1), a water soluble polyphenolic acid extracted from dried radix Salvia miltiorrhiza Bunge, which is an important part of Chinese folk medicine. Over 20 Salvia species have been used in traditional Chinese folk medicine for the treatment of coronary heart diseases and strokes. According to Li et al. [20] only the official species of $S$. miltiorrhiza Bunge meet the requirements set forth and ascribed as the formal traditional medicinal plant Danshen.

SA-B has therapeutic potential against different medical conditions [21-23]. SA-B has been used in a clinical trial and has not displayed any obvious side effects [24].

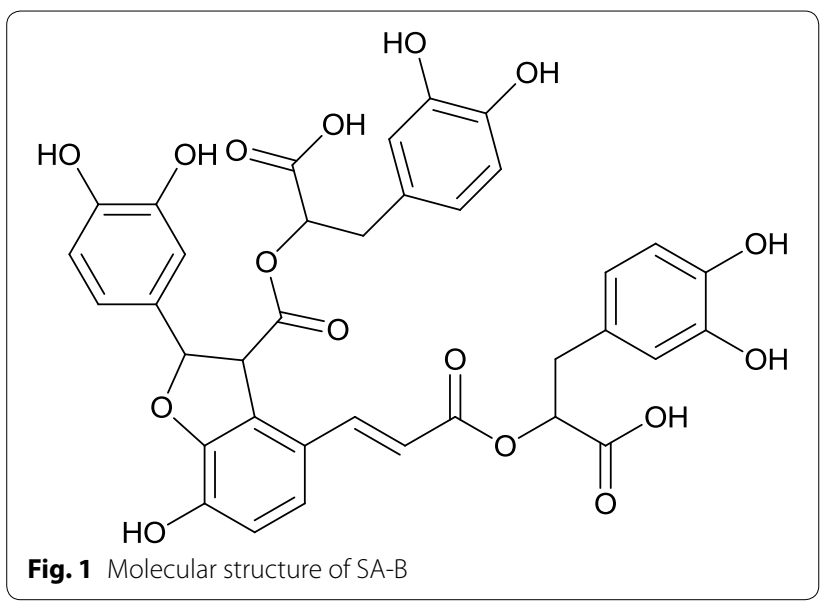

To our knowledge this is the first report on anti-infective activity of SA-B and the first report on anti-adhesion activity of Chinese herbs against $N$. meningitis.

\section{Methods \\ Materials}

Biomax Ultrafree-15 centrifugal filter devises with $100 \mathrm{kDa}$ cut offs were obtained from Millipore. D-biotinoyl- $\varepsilon$-aminocaproic acid-N-hydroxysuccinimide ester, streptavidin-POD conjugate, and ABTS-substrate were purchased from Roche diagnostics. Bovine thyroglobulin was obtained from Sigma. Polyvinylchloride microtiter plates (Falcon Flexible Plate) were from Becton-Dickinson Labware. Bacterial culture plates were purchased from Sarstedt and GCB agar from Oxoid Ltd. High-glucose DMEM cell culture medium, fetal bovine serum (FBS), L-glutamine, trypsin-EDTA solution and sterile phosphate buffered saline (PBS) for cell culture were from Gibco and cell culture plates from Greiner. Nitrocellulose membranes (Protran ${ }^{\circledR}$ ) were obtained from Schleicher \& Schuell BioScience. Super Signal solution was from Pierce and X-ray Hyperfilm ${ }^{\mathrm{TM}}$ from Amersham Pharmacia Biotech.

\section{Salvianolic acid B samples}

Purified radix extract SA-B (98\% purity) was purchased from Ivy Fine Chemicals. SA-B extract of $70 \%$ purity was purified from the radix $S$. miltiorrhizae by using ethanol extraction followed by column chromatography according to the method of Fung et al., 1993 [25]. Shortly: $500 \mathrm{~g}$ of S. miltiorrhiza Bunge was heated with $50 \%$ ethanol (3 L) for $4 \mathrm{~h}$. The mixture was allowed to cool down, after which it was filtered. After evaporation of ethanol the resulting aqueous solution was concentrated and allowed to stand overnight. After filtration, the aqueous solution was freeze-dried to a powder-like product (35 g). Column chromatography was then carried out by using $\mathrm{C}_{18}$ reverse phase column (Alltech, USA) with solvent gradient water to $80 \%$ aqueous methanol. The separation was monitored by thin layer chromatography on silica gel (Merck, Germany) with solvent system n-butanol/acetic acid $/ \mathrm{H}_{2} \mathrm{O}$ and the purity was determined by using HPLC chromatography on $\mathrm{C}_{18}$ reverse phase column (Ultrasphere-ODS from Beckman, USA with $20 \%$ aqueous $\mathrm{CH}_{3} \mathrm{CN}$, UV 254 detector).

Initially both SA-B samples were tested for the binding inhibition activity against $N$. meningitidis. All the other experiments were carried out by using the SA-B of $70 \%$ purity.

\section{Bacterial strain and culture conditions}

Neisseria meningitidis serogroup C class I strain 8013 (X. Nassif, INSERM U570, Paris, France) was cultured at 
$37^{\circ} \mathrm{C}$ in $\mathrm{CO}_{2}$ atmosphere for $18 \mathrm{~h}$ on GCB agar containing supplements described by Kellogg et al. [26].

\section{Isolation and biotin labelling of meningococcal pili}

Isolation and biotin labelling of pili were carried out as described before $[5,6]$. N. meningitidis bacteria were harvested from five plates and suspended into $20 \mathrm{~mL}$ of ice cold and sterile Hepes buffer $(10 \mathrm{mmol} / \mathrm{L})$. The suspension was mixed vigorously for $30 \mathrm{~s}$ and centrifuged $(20 \mathrm{~min}$ at $4{ }^{\circ} \mathrm{C}, 8000 \mathrm{~g}$ ). The supernatant was applied to a Biomax Ultrafree-15 centrifugal filter device (cut off $100 \mathrm{kDa}$ ) and centrifuged $\left(\right.$ at $\left.4{ }^{\circ} \mathrm{C}, 1000 \mathrm{~g}\right)$. The concentrate was washed twice with $15 \mathrm{~mL}$ of Hepes buffer $(10 \mathrm{mmol} / \mathrm{L})$ and centrifuged as described above, to the volume of $1 \mathrm{~mL}$. The concentrate was collected and stored at $4{ }^{\circ} \mathrm{C}$. Biotin labelling of the isolated pili was performed in PBS by using D-biotinoyl- $\varepsilon$-aminocaproic acid-N-hydroxysuccinimide ester according to the instructions of the manufacturer. The biotin-labelled pili were stored at $4{ }^{\circ} \mathrm{C}$.

\section{Inhibition of pili binding to bovine thyroglobulin}

The inhibition of meningococcal pili binding to bovine thyroglobulin was tested as described before by using dry milk powder as a background control [6]. Aliquots of $100 \mu \mathrm{L}$ of thyroglobulin $(0.1 \mathrm{mg} / \mathrm{mL})$ were incubated on polyvinylchloride microtiter plates at $4{ }^{\circ} \mathrm{C}$ overnight. The wells were washed five times with washing buffer $[0.05 \%(\mathrm{v} / \mathrm{v})$ Tween 20 in PBS, pH 7.4]. The non-specific binding sites were blocked by dry milk powder solution [5\% (w/v) dry milk powder, $0.05 \%(\mathrm{v} / \mathrm{v})$ Tween 20 in PBS, pH 7.4], $250 \mu \mathrm{L} /$ well. The plates were incubated for $1 \mathrm{~h}$ at room temperature. Biotin labelled pili were diluted 1:4 in PBS. Both of the SA-B samples were diluted with water to the concentration of $0.625 \mathrm{mg} / \mathrm{mL}$, serially diluted down to the concentration of $0.00125 \mathrm{mg} / \mathrm{mL}$ and dilutions were separately mixed 1:1 with biotin-labelled pili. Pili-sample mixtures were incubated at room temperature for $1 \mathrm{~h}$ on a gentle rocking platform. Biotin labelled pili diluted 1:8 in PBS without herbal extract addition was treated identically to samples and used as a control. Then, $100 \mu \mathrm{L}$ of the pre incubated solutions were added to washed, thyroglobulin-coated microtiter plate wells. After $1 \mathrm{~h}$ incubation at room temperature the wells were washed five times as described above, followed by Streptavidin-POD addition $[100 \mu \mathrm{L}$ of StreptavidinPOD conjugate in dry milk powder solution (1:4000)] and by $1 \mathrm{~h}$ incubation at room temperature. After five washes with washing buffer, an aliquot of $100 \mu \mathrm{L}$ of ABTS-substrate was added. The absorbance was measured at $405 \mathrm{~nm}$. The assays were always carried out in triplicates.

\section{Dot binding inhibition assay}

Human epithelial HEC-1B cell line, a cell culture model for $N$. meningitidis $[9,19]$ was obtained from Xavier
Nassif (INSERM U570, Paris, France). Cells were cultured on cell culture dishes in high-glucose DMEM supplemented with $10 \%$ heat-inactivated FBS and $4 \mathrm{mM}$ L-glutamine at $37{ }^{\circ} \mathrm{C}$ with $5 \% \mathrm{CO}_{2}$, without an addition of antibiotics. Cells from passages between 11 and 14 were harvested using a diluted trypsin-EDTA solution and centrifuged at $400 \mathrm{~g}, 4 \mathrm{~min}$. Cells were washed twice with ice cold sterile PBS, centrifuged as described above and stored at $-20^{\circ} \mathrm{C}$ until used.

In dot binding inhibition assay the anti-adhesion activity of SA-B was tested against meningococcal pili binding to HEC-1B cells. HEC-1B cells were collected from one plate as described above. They were suspended in $200 \mu \mathrm{L}$ of PBS and diluted 1:10 with PBS. Cell suspensions of $2 \mu \mathrm{L}$ were pipetted as dots on a nitrocellulose membrane and allowed to dry. Dry milk powder solution [5\% (w/v) dry milk powder, $0.05 \%(\mathrm{v} / \mathrm{v})$ Tween 20 in PBS, pH 7.4] was used to block the non-specific binding sites. After two hours of incubation (at room temperature) with gentle rocking on the rocking platform, the membrane was washed three times with washing buffer $[0.05 \%(\mathrm{v} / \mathrm{v})$ Tween 20 in PBS, $\mathrm{pH}$ 7.4]. The $70 \%$ SA-B sample was diluted with water to the concentration of $1 \mathrm{mg} / \mathrm{mL}$. The diluted sample and biotin labelled diluted pili (1:10 in dry milk powder solution) were mixed $1: 1$ and preincubated for $1 \mathrm{~h}$ at room temperature. Labelled pili-sample mixture was added into the washed membrane and the incubation was performed for $90 \mathrm{~min}$ at room temperature. Biotin labelled pili dilution without herbal extract addition was treated identically to the sample and used as a positive control. After three washes with washing buffer, Streptavidin-POD conjugate diluted in dry milk powder solution (1:4000) was added into the membrane. The membrane was incubated for $1 \mathrm{~h}$ at room temperature. Finally, the membrane was washed and Super Signal Solution was added. The membrane was exposed to Hyperfilm ${ }^{\mathrm{TM}}$ and developed.

\section{Antimicrobial assay on microtiter plates}

Antimicrobial activity of SA-B was tested against $N$. meningitidis. Overnight plate-cultured $N$. meningitidis bacteria were suspended in cold GC broth to an optical density of 0.180 at $550 \mathrm{~nm}$. The corresponding colony forming unit (CFU) was $10^{8}$. Diluted bacterial suspension $(90 \mu \mathrm{L})$ and SA-B dilutions ranging from 2 to $0.03 \mathrm{mg} /$ $\mathrm{mL}(10 \mu \mathrm{L})$ were incubated on microtiter plate at $37^{\circ} \mathrm{C}$, in $\mathrm{CO}_{2}$ atmosphere, for $2 \mathrm{~h}$. As a control, bacteria were incubated in the absence of SA-B or with ampicillin $(0.1 \mathrm{mg} / \mathrm{mL})$. The antimicrobial activity was analysed by plating the incubation mixtures in triplicate on GCB-agar plates. The survived colony forming units were counted next day. The growth inhibition was analysed by comparing the CFUs of bacteria-sample -mixture and control bacteria. 


\section{Statistical analysis}

Results for the inhibition assay were reported as mean \pm standard deviation (SD). Two-tailed, unpaired Student's t test (Microsoft Excel 2007, Microsoft Corp., Santa Rosa, CA, USA) was used for calculating the significance of the binding inhibition for both SA-B of $70 \%$ purity and SA-B of $98 \%$ purity compared to control, and for calculating the differences between inhibition caused by SA-B of $70 \%$ purity and by SA-B of $98 \%$ purity. The significance was defined as values of $\mathrm{P}<0.05$. Results for the antimicrobial assay were reported as mean \pm standard deviation (SD). Two-tailed, unpaired Student's t test (Microsoft Excel 2007, Microsoft Corp., Santa Rosa, CA, USA) was used for calculating the significance of the differences between CFUs from antimicrobial tests and control bacteria. Significance was defined as a value of $\mathrm{P}<0.001$.

\section{Results}

\section{Anti-adhesive activity of the SA-B extracts}

To demonstrate the SA-B anti-adhesive activity, both SA-B of $98 \%$ purity and SA-B of $70 \%$ purity were tested. The binding inhibition for both SA-B of $70 \%$ purity $(\mathrm{P}<0.001)$ and SA-B of $98 \%$ purity $(\mathrm{P}<0.001)$ was significant compared to control. Binding inhibition activity of the SA-B with $98 \%$ purity was $78 \%$ at the concentration of $0.3 \mathrm{mg} / \mathrm{mL}$, and concentration for the $50 \%$ inhibition was $2.4 \mu \mathrm{g} / \mathrm{mL}$. The SA-B extract of $70 \%$ purity inhibited the pili binding almost totally (93\%) at the concentration of $0.3 \mathrm{mg} / \mathrm{mL}$ and $50 \%$ inhibitory activity was achieved at the concentration of $0.6 \mu \mathrm{g} / \mathrm{mL}$. Quite similar results in the binding inhibition activity were achieved with both extracts, however the SA-B of $70 \%$ purity was significantly $(P=0.03)$ more efficient in binding inhibition than SA-B of $98 \%$ purity (Fig. 2). In the lowest concentrations (below $0.01 \mathrm{mg} / \mathrm{mL}$ ) the difference was non-existent, except at the concentration of $0.002 \mathrm{mg} / \mathrm{mL}(\mathrm{P}=0.01)$. When the $70 \% \mathrm{SA}-\mathrm{B}(0.5 \mathrm{mg} /$ $\mathrm{mL}$ ) extract was tested for the inhibition of meningococcal pili binding to HEC-1B epithelial cells, the extract was able to completely inhibit the attachment of meningococcal pili (Fig. 3).

\section{Antimicrobial activity of the SA-B}

Antimicrobial activity has been found amongst berries, herbs and berry phenolics [27-29]. In antimicrobial assay the growth of $N$. meningitidis was inhibited effectively $(\mathrm{P}<0.001)$ in relatively high concentrations $(2 \mathrm{mg} /$

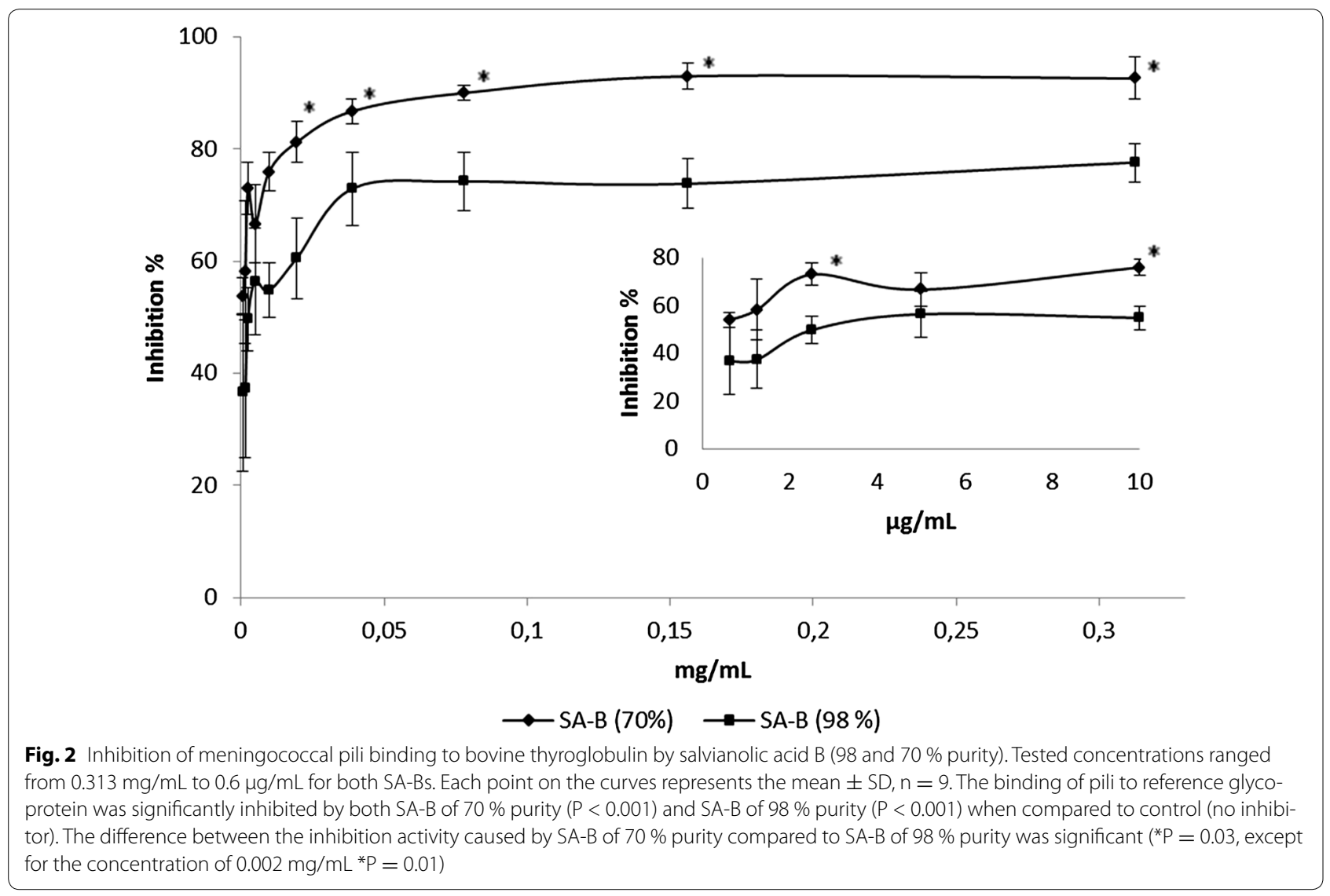




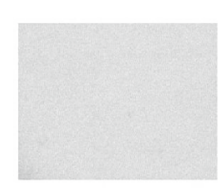

SA-B

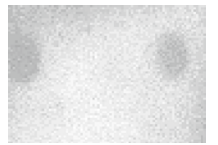

Positive control

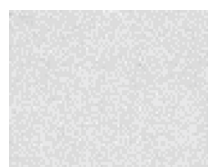

Control A

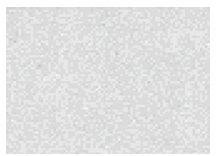

Control B
Fig. 3 Inhibition of meningococcal pili binding to HEC-1B human epithelial cells by SA-B. Positive control shows pili biding without presence of SA-B. Controls A and B do not contain pili or Streptavidin$P O D$, respectively

$\mathrm{mL}$ ) of the $70 \%$ SA-B. Other tested SA-B concentrations (1-0.03 $\mathrm{mg} / \mathrm{mL})$ did not have significant effect on meningococcal growth and the bacterial survival was found to be between 58 and $89 \%$, respectively. Bacterial survival with ampicillin was $23 \%$ (Fig. 4).

\section{Discussion}

Even if the herbal solution is efficacious, immediate determination of the active substances may not be possible, particularly in herbal solutions that contains many compounds. Components in a crude extract could act synergistically, have unknown interactions and interact to diminish possible adverse effects of the components. Also, if used properly, a mixture of several crude extracts could have greater beneficial effects compared to a single plant extract. This has been shown by using Chinese herbs against hemagglutination and adhesion of Escherchia coli [30].

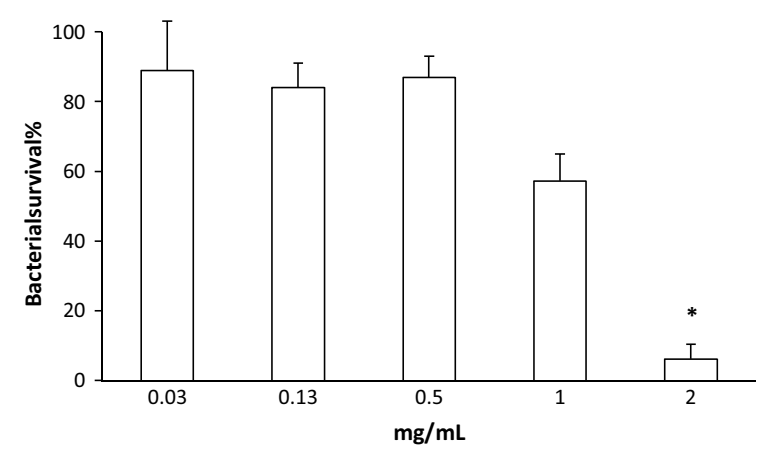

Fig. 4 Antimicrobial activity of SA-B against Neisseria meningitidis. Bacterial survival compared with control, mean \pm SD of three experiments. ${ }^{*} \mathrm{P}<0.001$ against the bacterial control
Neisseria meningitidis adhesion is mediating by type 4 pili, which carries two main potential adhesins [31, 32], but the adhesion mechanisms are complicated [33]. The SA-B sample of $70 \%$ purity may contain components that interact with different binding activities of N. meningitidis. This may be one explanation for higher binding inhibitory activity of $70 \%$ SA-B compared to $98 \%$ SA-B. Our previous results suggest carbohydrate recognition for $N$. meningitidis and binding inhibition by milk oligosaccharides [5]. Parallel to results achieved here we have also shown that berry extracts rich in polyphenols are active against pili binding $[6,9]$.

Bacterial binding to host tissues is an important phase before bacterial infections $[1,2]$. In this study we found binding inhibition activity of SA-B against meningococcal pili, which mediate $N$. meningitidis adhesion. There is a lack of proper vaccination against $N$. meningitidis [17]. Natural products as anti-adhesives could offer an important solution for prevention. As an anti-adhesive agent SA-B could possibly reduce the number of nasopharyngeal carries and thus reduce the risk of spreading the infection. The use of known, natural nutritional products in a form of an extract rather than a purified compound would be practical and economic for prevention purposes. This could be useful especially in developing countries, where proper preservation of vaccines is limited and epidemics of meningitis are a major health problem.

Here we show the inti-infective, anti-adhesive effect of SA-B. In anti-adhesion therapy the interaction between the pathogen and the host is inhibited without killing the bacteria thus avoiding rise of bacterial resistance [34]. Antimicrobial activity of the SA-B was only found in higher and not in lower concentrations, and in concentrations possessing binding inhibitory activity. The inhibitory activity found in the present study was not antimicrobial but anti-adhesive indicating SA-B as a candidate for anti-adhesion therapy against $N$. meningitidis.

\section{Conclusions}

Our results indicate that both 70 and $98 \%$ pure SA-B extracts may prevent meningococcal infections by inhibiting meningococcal binding to host cells. It can be assumed that this inhibition may also have a reducing impact on the amount of nasopharyngeal carriers of $N$. meningitidis and thus preventing the spread of meningococcal infections between humans. One could conclude that SA-B and its source, dried radix Salviae miltiorrhizae, an important part of Chinese folk medicine, could be valuable candidates for further research in meningococcal disease prevention.

Abbreviations

CFU: colony forming unit; SA-B: salvianolic acid B. 


\section{Authors' contributions}

SH, MT and CTK conceived and designed the study. SH performed the experiments and MT carried out the statistical analysis. CL purified SA-B from the radix. $\mathrm{SH}$ and CTK wrote the manuscript. All authors read and approved the manuscript.

\section{Author details}

1 School of Pharmacy, University of Eastern Finland, Kuopio Campus, Kuopio, Finland. ${ }^{2}$ Institute of Public Health and Clinical Nutrition, University of Eastern Finland, Kuopio Campus, Kuopio, Finland. ${ }^{3}$ Shanghai University of Traditional Chinese Medicine, Shanghai, China. ${ }^{4}$ Ruralia Institute, University of Helsinki, Lönnrotinkatu 7, 50170 Mikkeli, Finland.

\section{Acknowledgements}

We thank Prof. Xavier Nassif for providing the bacterial strain and the cell line. The excellent technical assistance of Sari Ukkonen is acknowledged. This work was supported by Regional Council of Pohjois-Savo.

\section{Competing interests}

The authors declare that they have no competing interests.

Received: 4 November 2015 Accepted: 4 January 2016

Published online: 13 January 2016

\section{References}

1. Ofek I, Hasty DL, Sharon N. Anti-adhesion therapy of bacterial diseases: prospects and problems. FEMS Immunol Med Microbiol. 2003;38:181-91.

2. Zopf D, Roth S. Oligosaccharide anti-infective agents. Lancet. 1996;347:1017-21.

3. Cozens D, Read RC. Anti-adhesion methods as novel therapeutics for bacterial infections. Expert Rev Anti Infect Ther. 2012;10:1457-68.

4. Idänpään-Heikkilä I, Simon PM, Zopf D, Vullo T, Cahill P, Sokol K, Tuomanen E. Oligosaccharides interfere with the establishment and progression of experimental pneumococcal pneumonia. J Infect Dis. 1997;176:704-12.

5. Hakkarainen J, Toivanen M, Leinonen A, Frängsmyr L, Strömberg N, Lapinjoki S, Nassif X, Tikkanen-Kaukanen C. Human and bovine milk oligosaccharides inhibit Neisseria meningitidis pili attachment in vitro. J Nutr. 2005;135:2445-8.

6. Toivanen M, Ryynänen A, Huttunen S, Duricova J, Riihinen K, Törrönen R, Lapinjoki S, Tikkanen-Kaukanen C. Binding of Neisseria meningitidis pili to berry polyphenolic fractions. J Agric Food Chem. 2009;57:3120-7.

7. Toivanen M, Huttunen S, Duricova J, Soininen P, Laatikainen R, Loimaranta $\checkmark$, Haataja S, Finne J, Lapinjoki S, Tikkanen-Kaukanen C. Screening of binding activity of Streptococcus pneumoniae, Streptococcus agalactiae and Streptococcus suis to berries and juices. Phytother Res. 2010;24(Suppl 1):95-101.

8. Huttunen S, Toivanen M, Arkko S, Ruponen M, Tikkanen-Kaukanen C. Inhibition activity of wild berry juice fractions against Streptococcus pneumoniae binding to human bronchial cells. Phytother Res. 2011;25:122-7.

9. Toivanen M, Huttunen S, Lapinjoki S, Tikkanen-Kaukanen C. Inhibition of adhesion of Neisseria meningitidis to human epithelial cells by berry juice polyphenolic fractions. Phytother Res. 2011;25:828-32.

10. Burger O, Ofek I, Tabak M, Weiss E, Sharon N, Neeman I. A high molecular mass constituent of cranberry juice inhibits Helicobacter pylori adhesion to human gastric mucus. FEMS Immunol Med Microbiol. 2000;29:295-301.

11. Lee JH, Park EK, Uhm CS, Chung MS, Kim KH. Inhibition of Helicobacter pylori adhesion to human gastric adenocarcinoma epithelial cells by acidic polysaccharides from Artemisia capillaris and Panax ginseng. Planta Med. 2004;70:615-9.

12. Grace MH, Guzman I, Roopchand DE, Moskal K, Cheng DM, Pogrebnyak $\mathrm{N}$, Raskin I, Howell A, Lila MA. Stable binding of alternative proteinenriched food matrices with concentrated cranberry bioflavonoids for functional food applications. J Agric Food Chem. 2013;61:6856-64.
13. Lee JH, Shim JS, Chung MS, Lim ST, Kim KH. In vitro anti-adhesive activity of green tea extract against pathogen adhesion. Phytother Res. 2009;23:460-6.

14. Lengsfeld C, Titgemeyer F, Faller G, Hensel A. Glycosylated compounds from okra inhibit adhesion of Helicobacter pylori to human gastric mucosa. J Agric Food Chem. 2004;52:1495-503.

15. Caugant DA, Maiden MCJ. Meningococcal carriage and disease-a population biology and evolution. Vaccine. 2009;27(Suppl 2):B64-70.

16. Nassif $X$. Interaction mechanisms of encapsulated meningococci with eukaryotic cells: what does this tell us about the crossing of the bloodbrain barrier by Neisseria meningitidis?. Curr Opin Microbiol. 1999:2:71-7.

17. Girard MP, Preziosi MP, Aguado MT, Kieny MP. A review of vaccine research and development: meningococcal disease. Vaccine. 2006;24:4692-700.

18. Nassif X, Lowy J, Stenberg P, O'Gaora P, Ganji A, So M. Antigenic variation of pilin regulates adhesion of Neisseria meningitidis to human epithelial cells. Mol Microbiol. 1993;8:719-25.

19. Nassif X, Pujol C, Morand P, Eugene E. Interactions of pathogenic Neisseria with host cells. Is it possible to assemble the puzzle? Mol Microbiol. 1999;32:1124-32.

20. Li MH, Chen JM, Peng Y, Wu Q, Xiao PG. Investigation of Danshen and related medicinal plants in China. J Ethnopharmacol. 2008;120:419-26.

21. Adams J, Wang R, Yang J, Lien E. Preclinical and clinical examinations of Salvia miltiorrhiza and its tanshinones in ischemic conditions. Chin Med. 2006;1:3.

22. Chor SY, Hui AY, To KF, Chan KK, Go YY, Chan HLY, Leung WK, Sung JJY. Anti-proliferative and pro-apoptotic effects of herbal medicine on hepatic stellate cell. J Ethnopharmacol. 2005;100:180-6.

23. Lin SL, Lee IT, Chen YH, Lin FY, Sheu LM, Ku HH, Shiao MS, Chen JW, Chen YL. Salvianolic acid B attenuates MMP-2 and MMP-9 expression in vivo in apolipoprotein-E-deficient mouse aorta and in vitro in LPS-treated human aortic smooth muscle cells. J Cell Biochem. 2007;100:372-84.

24. Liu P, Hu YY, Liu C, Zhu DY, Xue HM, Xu ZQ, Xu LM, Liu CH, Gu H, Zhang ZQ. Clinical observation of salvianolic acid B in treatment of liver fibrosis in hepatitis B. World J Gastroenterol. 2002;8:679-85.

25. Fung KP, Zeng LH, Wu J, Wong HN, Lee CM, Hon PM, Chang HM, Wu TW. Demonstration of the myocardial salvage effect of lithospermic acid $B$ isolated from the aqueous extract of Salvia miltiorrhiza. Life Sci. 1993;52:239-44

26. Kellogg DS, Peacock WL, Deacon WE, Brown L, Pirkle DI. Neisseria gonorrhoeae I. Virulence genetically linked to clonal variation. J Bacteriol. 1963;85:1274-9.

27. Cavanagh HMA, Hipwell M, Wilkinson JM. Antibacterial activity of berry fruits used for culinary purposes. J Med Food. 2003;6:57-61.

28. Ooi LS, Li Y, Kam SL, Wang H, Wong EY, Ooi VE. Antimicrobial activities of cinnamon oil and cinnamaldehyde from the Chinese medicinal herb Cinnamomum cassia Blume. Am J Chin Med. 2006;34:511-22.

29. Puupponen-Pimiä R, Nohynek L, Hartmann-Schmidlin S, Kähkönen M, Heinonen M, Määttä-Riihinen K, Oksman-Caldentey KM. Berry phenolics selectively inhibit the growth of intestinal pathogens. J Appl Microbiol. 2005;98:991-1000.

30. Tong YQ, Wu Q, Zhao DK, Liu Y, Cao M, Zhang L, Zeng S. Effects of Chinese herbs on the hemagglutination and adhesion of Escherichia coli. Afr J Tradit Complement Altern Med. 2011:8:82-7.

31. Nassif X, So M. Interaction of pathogenic Neisseriae with nonphagocytic cells. Clin Microbiol Rev. 1995;8:376-88.

32. Rudel T, Scheuerpflug I, Meyer TF. Neisseria PilC protein identified as type-4 pilus tip-located adhesin. Nature. 1995;373:357-9.

33. Miller F, Phan G, Brissac T, Bouchiat C, Lioux G, Nassif X, Coureuil M. The hypervariable region of meningococcal major pilin PilE controls the host cell response via antigenic variation. M Bio. 2014. doi:10.1128/ mBio.01024-13.

34. Joosten JAF, Loimaranta V, Appeldoorn CCM, Haataja S, El Maate FA Liskamp RMJ, Finne J, Pieters RJ. Inhibition of Streptococcus suis adhesion by dendritic galabiose compounds at low nanomolar concentration. J Med Chem. 2004;47:6499-508. 